\title{
Ontology Population Reusing Resources for Dialogue Intent Detection: Generic and Multilingual Approach
}

\author{
Cristina Aceta, Izaskun Fernández \\ TEKNIKER \\ Basque Research and Technology Alliance \\ Eibar, Spain \\ cristina. aceta@tekniker.es \\ izaskun. fernandezltekniker.es
}

\author{
Aitor Soroa \\ CCIA Group \\ University of the Basque Country \\ San Sebastián, Spain \\ a.soroadehu. eus
}

\begin{abstract}
This work presents a generic semi-automatic strategy to populate the domain ontology of an ontology-driven task-oriented dialogue system, with the aim of performing successful intent detection in the dialogue process, reusing already existing multilingual resources. This semi-automatic approach allows ontology engineers to exploit available resources so as to associate the potential situations in the use case to FrameNet frames and obtain the relevant lexical units associated to them in the target language, following lexical and semantic criteria, without linguistic expert knowledge. This strategy has been validated and evaluated in two use cases, from industrial scenarios, for interaction in Spanish with a guide robot and with a Computerized Maintenance Management System (CMMS). In both cases, this method has allowed the ontology engineer to instantiate the domain ontology with the intentrelevant information with quality data in a simple and low-resource-consuming manner.
\end{abstract}

\section{Introduction}

Nowadays, the arrival of new technologies and virtual assistants (e.g., Siri, Alexa) prove that naturally interacting with different devices and applications is a reality that is constantly improving through time. In many contexts, such as industrial scenarios, this fact has contributed to an increasing demand of technologies that allow workers to naturally communicate (i.e, without relying on specific constructions or keywords) with industrial systems such as robots, machines or information systems so as to increase productivity and security (GonzálezDocasal et al., 2020), among other aspects.

A common approach to support natural communication between humans and machines are dialogue systems. When the goal is for the system to perform a specific action requested by the user, task-based dialogue systems are used (Jurafsky and
Martin, 2020). As stated in Jurafsky and Martin (2020), a wide range of modern commercial taskoriented dialogue systems are to some extent inspired in the Genial Understander System (GUS) architecture in Bobrow et al. (1977). This architecture consists of frame structures, that model the situations to be invoked by the user -the intentsand the required slots -arguments- to be provided for each frame. To determine the frame to be selected for a specific request, an intent detection step is needed.

The main research goal is to develop a generic semantic-based task-oriented dialogue system inspired by GUS, in which both the dialogue process and the domain modelling are conceptualized in a generic manner by making use of ontologies ${ }^{1}$. The focus in this paper is the population process of the domain ontology to efficiently perform intent detection, minimizing human intervention and linguistic knowledge when adapting the dialogue system to specific use cases.

Communication based on natural language can use a variety of words and expressions to convey the same meaning. Therefore, a dialogue system that interacts with users must be able to recognize different words that evoke the same situation or refer to the same entity to perform successful intent detection $^{2}$. In an ontology-based approach, to populate the domain ontology with this information, it is very important that the maximum number of these possibilities is considered. Doing so manually would be a high time- and resource-consuming task, and the tendency is to apply semi-automatic or automatic techniques (Makki, 2017; Benabbas et al., 2018). According to this tendency, this work presents a generic strategy that aims to overcome

\footnotetext{
${ }^{1}$ The former is usually called dialogue ontology, and the latter domain ontology.

${ }^{2}$ For the previous example, besides inform, the verb tell may also elicit the give information intent.
} 
the challenge of instantiating the domain ontology with intents and their trigger words by semiautomatically exploiting existing lexical resources, without reducing the quality of the results and decreasing time and costs in the adaptation to different scenarios and applications. The result, thus, is a semi-automatic process which leverages existent multilingual lexical resources to (1) be able to model the intents specific to a use case by using existing Semantic Frames (Fillmore et al., 1976) and (2) obtain the maximum number of words that trigger them in different languages -i.e., their translation equivalents-, so as to instantiate the domain ontology of the dialogue system approach previously described. This process has been validated in two use cases from industrial scenarios.

The rest of the paper is organised as follows: Section 2 will provide relevant Related Work. Section 3 will describe the ontology population strategy. Section 4 will present the validation and evaluation of the strategy through 2 use cases, and Section 5 will provide Conclusions and Further Work.

\section{Related Work}

There is a wide range of works in the literature that deal with intent detection, as it is one of the core tasks in the interpretation component in taskoriented dialogue systems (Gupta et al., 2019). In this sense, two main approaches to perform intent detection can be distinguished: on the one hand, approaches that rely on machine learning (ML) methods and, on the other hand, approaches that make use of ontologies. For both methodologies, a fair amount of data is needed and, thus, cannot cope with scenarios with limited or nonexistent labeled data, which implies the necessity of performing manual work, which is resource-consuming (Chen et al., 2017).

For machine-learning-based methods, several approaches can be observed in the literature that make use of traditional ML algorithms such as Support Vector Machines (Cortes and Vapnik, 1995) or logistic regression (Bishop, 2006). However, modern approaches employ deep learning (DL) methods (Louvan and Magnini, 2020; Chen et al., 2019). As mentioned previously, ML-based approaches require of a fair amount of data to perform training tasks, especially DL models.

For ontology-based methods, the population of the domain ontology to perform intent detection is generally based on patterns, which can be obtained automatically or semi-automatically. The formalization in Cassier et al. (2019), who have designed an intent detection system for emails in French, is inspired in semantic frames, and the population of the domain ontology is based on a set of mainly automatically-generated patterns that allow to determine the words that trigger the intents. However, to obtain these patterns, a large amount of data was collected and processed. On the other hand, Quamar et al. (2020) present a dialogue system in which intent detection in health-related texts is performed. As in the previous case, the authors also rely on patterns to determine Key and Dependent concepts, which are considered to determine the intent in a sentence. Also, the information related to patterns is obtained combining expert knowledge and automatic methods.

Considering the previous remarks, intent detection seems to require either a large volume of data or manual work. In ontology-based approaches, for the former, the task of instantiating the domain ontology in low-resourced scenarios is severely limited and, for the latter, the use of manual expert knowledge is time and resource consuming. In this sense, a well-known challenge to the Semantic Web community is how to reduce the amount of manual work in the ontology population task. As noted by Kontopoulos et al. (2017), most approaches for ontology population make use of textual input and rely on natural language processing techniques to obtain the necessary knowledge to populate the ontology (Corcoglioniti et al., 2016; Makki, 2017), whereas approaches that rely on data that is structured at some degree -which are of interest for this work- are less common (Leshcheva et al., 2017; Kontopoulos et al., 2017). Considering this, the authors in Kontopoulos et al. (2017) use structured knowledge in Linked Data for ontology population.

The approach presented in this paper aims to overcome these challenges providing a methodology that could be used in scenarios with a lack of data with a relative minimal effort, without depending of expert linguistic work, and by making use of already available structured knowledge.

\subsection{Resources}

The concept of FrameNet originates from Frame Semantics (Fillmore et al., 1976), which is the linguistic theory that asserts that specific words evoke specific frames. In a nutshell, FrameNet constitutes a multilingual predicate resource that aims 
to model situations (frames) and the words that elicit them (lexical units) in a comprehensive way, and also provides with the necessary actors that take part on them (Frame Elements) as a sort of a slot-modelling approach. In FrameNet, frames are not language-specific, and therefore are common in all language versions. That said, the data that varies across languages are, for each frame, the corresponding lexical units in the target language.

FrameNet, however, is not the only resource that provides predicate-related information in the literature. Other examples are VerbNet (Schuler, 2006) or PropBank (Kingsbury and Palmer, 2003). Each of these resources present characteristics that the rest of alternatives do not offer which, in the end, has generated the need to integrate them into a single repository. The result of this integration is Predicate Matrix (PM) (De Lacalle et al., 2014), in which data from different predicate (e.g. English FrameNet), lexical (e.g., WordNet (Miller, 1995)) and semantic (e.g. Suggested Upper Merged Ontology - SUMO (Pease et al., 2002)) resources is mapped, providing a "multilingual interoperable predicate lexicon" (De Lacalle et al., 2016).

Regarding the data integrated in PM, one of the lexical resources included is WordNet. WordNet (Miller, 1995) is a lexical knowledge base that stores nouns, verbs, adjectives and adverbs -and their corresponding senses- and groups them semantically into what are called synsets, which are described in Miller (1995) as "sets of cognitive synonyms". In this sense, words are interlinked in semantic terms, considering each word's senses. WordNet has been adapted to many languages and even some wordnets have been integrated into single resources, such as the Multilingual Central Repository-MCR-(Atserias et al., 2004). The MCR, which at the time of writing this paper is on version 3.0, aims to provide a powerful and rich multilingual lexical knowledge base (Atserias et al., 2004). This lexical knowledge base integrates data from multiple resources, including WordNet -in its 3.0 version- in six different language versions (English, Spanish, Catalan, Basque, Galician and Portuguese). Moreover, following the EuroWordNet architecture (Atserias et al., 2004), the MCR interconnects these wordnets using interlingual indices (ILI), also based in WordNet 3.0, for equivalent synsets in different languages. Furthermore, the repository has been enriched with ontological knowledge coming from semantic resources such as Base Level Concepts (Rosch, 1977), WordNet domains (Bentivogli et al., 2004) and SUMO ${ }^{3}$ (Pease et al., 2002; Guinovart et al., 2021). MCR synsets are also mapped in PM.

In terms of semantic data, among others, PM includes SUMO. SUMO is intended as an upper level ontology to serve as a "foundation for more specific domain ontologies" (Niles and Pease, 2001) by including a wide range of general-purpose terms that comprehensively cover different fields, such as Linguistics, Computer Science or Artificial Intelligence. Also, these terms are formally defined through axioms, which makes SUMO a specially enriched resource. Thus, the aim of SUMO is to provide a comprehensive, precisely defined term ontology. The mapping of SUMO tags with the rest of the elements in PM allows to establish a categorization between the different terms in it.

It is worth noting that PM is centred in verbal information. Thus, for resources that account for multiple parts of speech (such as nouns or adjectives in WordNet or frames that have nominal lexical units in FrameNet), only verbal data is integrated, so as to be able to properly map the data from all the resources involved.

\section{Generic Strategy for Ontology Population for Intent Detection}

The aim of the generic strategy is to support ontologists in the process of task-oriented dialogue system domain ontology population, minimizing the manual work, reusing linguistic resources, but without expert knowledge of the linguistic field.

The core linguistic resource used for collecting the necessary information for the ontology population process is PM, since it integrates and maps, among others, the information contained in FrameNet, SUMO and the MCR-the resources that include most of the relevant data for this work.

The strategy will guide the ontologist through the selection of the relevant information within PM, and will gather, semi-automatically, the relevant intents (through frames), the associated trigger words (through lexical units) and their corresponding synonyms for a specific use case in which the dialogue will take place. Considering this, the main steps of the strategy are the following:

0. Use Case Characterization. The identification of the different situations (associated

\footnotetext{
${ }^{3}$ Equivalence ("=”), subsumption (“+”) and instantiation (“@”) mapping relations (Alvez et al., 2019).
} 
to intents) that surround the use case is performed. For example, for a guide robot, the situations involved may be to be taken to a given place or just to present information.

1. Linguistic-resource-driven data selection. For the given situations identified for the use case, a data selection from linguistic resources is performed, following three phases: frame selection, lexical unit selection and semantic extension and filtering.

2. Automatic data gathering for intents. Considering the selected data, the relevant knowledge to the intents is automatically gathered and structured in terms of the intents, their corresponding frames and the words that evoke them that are relevant to the use case. Up to this point, the gathered information is agnostic to the structure of the target domain ontology.

3. Query generation and ontology population. Once the relevant intent-related information is automatically obtained, the query for the final population of the target domain ontology will be generated.

The following sections will detail the aforementioned steps ${ }^{4}$.

\subsection{Linguistic-resource-driven data selection}

This first step is aimed to obtain the necessary information that will serve as input for the automatic data gathering step. This data will be selected according to the situations identified for the use case.

\subsubsection{Frame Selection}

The first stage in the process is to select the most suitable frames for the previously defined events in the use case. For this step, the ontology engineer will choose the relevant frames for the use case from the list of available FrameNet frames in PM.

For example, in a guide robot scenario described in more detail in Section 4.1-, one frame to be chosen would be Motion.

\subsubsection{Lexical Unit Selection}

For each selected frame, a set of lexical units in English are automatically extracted from PM and presented to the ontology engineer, who will select

\footnotetext{
${ }^{4}$ Step 0 has already been properly described and will not be included in next sections.
}

the relevant lexical units according to the use case. This step is necessary because it is not evident that all lexical units are suitable to all the cases the chosen frame may apply to.

To illustrate this with an example, the frame chosen in the previous section, Motion, applied to the guide robot use case, in which a robot may be asked to guide the user to some final destination, has the lexical units fn:blow.v, fn:fly.v, fn:go.v, fn:move.v, etc. associated in PM. Within the use case, some lexical units such as fn:fly.v do not apply, and must therefore not be selected. In this case, the relevant lexical units to be selected are go.v and move.v.

\subsubsection{Semantic Extension and Filtering}

The objective of this step is to extend semantically the previously selected lexical units by exploiting MCR synsets and SUMO tags.

For that, firstly, an automatic semantic extension is performed through the links from lexical units to MCR synsets in PM, collecting all the synsets related to given lexical units. However, not all the synsets in MCR for a lexical unit may apply to the use case, because polysemous words may acquire different meanings in a same frame, so a selection is required. For example, go may imply movement from one place to another or to pass away.

In PM, synsets are mapped to SUMO tags, which aim to group different synsets into single concepts. Since SUMO tags are more human-readable than synsets and the amount of tags is considerably lower without losing significance, the selection of synsets is proposed to be performed through SUMO tags. For that, the associated SUMO tags for the whole synset list are automatically extracted from PM and presented to the ontology engineer for the final selection.

To continue with the previous example, for fn:go.v, the mcr:SubjectiveAssessmentAttribute, mcr:OccupationalRole; duration, mcr:Motion and $m c r$ :Death SUMO tags are presented to the ontology engineer, from which they will select the most relevant ones for the use case (all except mcr:Death).

\subsection{Automatic Data Gathering for Intents}

The objective of this step is to obtain the final words in the target language that evoke the desired intents. For this, the translation equivalents corresponding to the set of synsets obtained through the data in the previous steps will be retrieved automatically. 
Taking as input the set of selected frames, lexical units and SUMO tags, the corresponding synsets will be automatically retrieved from PM. After that, the words in the target language that belong to these synsets will be obtained from the MCR, also automatically. As a result of this step, a set of synonyms in the target language(s) for each lexical unit will be obtained.

In the example used previously, for the frame Motion and the relevant synsets for the lexical unit go.v, the resulting synonyms in Spanish are the following: acudir, desplazarse, ir, mover, moverse, viajar and partir ${ }^{5}$.

At this point, all the necessary data to support intent detection has been obtained.

\subsection{Query Generation and Ontology Population}

In this step, the SPARQL query necessary to populate the ontology with the gathered data is created according to the modelling of the target ontology. To summarise, Figure 1 represents the complete process, including the example use case shown in the previous sections, which is described in more detail in Section 4.1.

\section{Validation and Evaluation}

The process has been implemented as an API REST and has been tested in two use cases: a guide robot scenario and a Computerized Maintenance Management System (CMMS), both for the Spanish language. The domain ontology used is the Domain Module for Task-Oriented Dialogue management Ontology (TODO) -TODODom- ${ }^{6}$, as it is an ontology that provides a top model for domain modelling for task-based dialogue systems. The implementation of the methodology to instantiate the ontology described in this work applied to these use cases will be detailed in the following lines.

\subsection{Use case \#1: Interacting with a Guide Robot in Spanish}

In this use case, the user needs to communicate to a guide robot in Spanish. This robot is able to guide the user to their destination of choice and to offer information from specific elements (a room, a machine, etc.). The situations that may be identified from this use case, thus, are: to move from one point to another, and to ask for information

\footnotetext{
${ }^{5}$ Attend, move, go, move, move, travel and leave

${ }^{6}$ https://w3id.org/todo/tododom
}

or details about something. After checking the FrameNet frames in PM, the ones deemed more relevant in this use case are the following: Motion, Taking and Arriving ${ }^{7}$.

The next step is to select the appropriate lexical units for each frame in PM. In this case, the total number of lexical units for all the frames selected in the previous step are 34. Although many of the lexical units do cover the use case, such as move and arrive, there are others, such as fly or blow, that are too specific and do not apply to the use case and must not be selected.

After this selection, the number of lexical units in English is reduced to 12: go, move (Motion); take (Taking); approach, arrive, come, enter, get, make, reach, return, visit (Arriving).

So as to semantically filter the synsets associated to each lexical unit, SUMO tags are used. In this case, the number of available SUMO tags is 34. In this use case, the application of semantic filtering is specially relevant, since from the initial 34 available tags, only two are applicable to the use case and have been selected: BodyMotion and Motion. The rest of tags were related to situations that were not considered in the use case, such as Death or Cooking and, thus, were not part of the final selection. This considerable reduction of tags reinforces the importance of this filtering step.

Finally, after having selected the frames, lexical units in English and SUMO tags, this information is used to automatically extract the applicable synsets and their translation equivalents in Spanish which, in this case, make a total of 32 . The vast majority of the synonyms are relevant to the use case (e.g., moverse, acudir, desplazarse, regresar ${ }^{8}$ ), although a very small portion of them are not entirely appropriate (e.g., estar_activo - to be active).

\subsection{Use case \#2: Interacting with a CMMS in Spanish}

In this use case, the target system is a Computerized Maintenance Management System (CMMS), in which the user should be able to ask about problem solving protocols, request blueprints and similar tasks. As the previous use case, the language used is Spanish. Considering the previous remarks, the

\footnotetext{
${ }^{7}$ Although the original FrameNet includes the frame Information, which would suit this use case, it is not included in PM due to the fact that it only has nominal lexical units. In the context of this work, this frame will remain out of the evaluation experiments carried out in Section 4.3.

${ }^{8}$ move, go, move and return, respectively.
} 


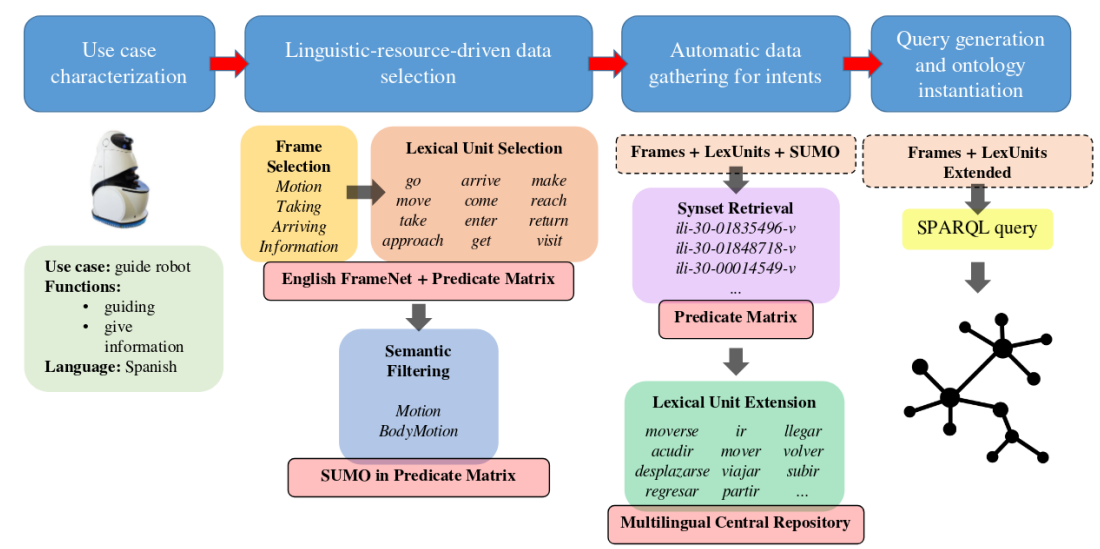

Figure 1: Diagram representing the ontology population process presented in this work in a guide robot scenario.

\begin{tabular}{lcc} 
& Guide & CMMS \\
\hline Gold & 25 & 17 \\
F & 667 & 137 \\
F+LU & 170 & 69 \\
F+SUMO & 139 & 70 \\
F+LU+SUMO & 32 & 39 \\
\hline
\end{tabular}

Table 1: Number of translation equivalents for each PM selected data configuration and use case.

identifiable situations in this use case are showing information and reporting and solving problems. The applicable frames are, thus, Resolve problem, Evidence, Reporting and Communication.

Regarding the lexical units, a total of 30 results have been obtained from the previously selected frames. After discarding some lexical units that were not applicable to the current use case, such as contradict or deal, the selected lexical units are the following: solve, resolve (Resolve problem); indicate, reveal, show (Evidence and Communication); inform, report, tell (Reporting).

In this next step, the number of remaining SUMO tags is 9 , which are reduced to Communication, VisualAttribute and IntentionalPsychologicalProcess. Given this selection, the total number of automatically obtained word synonyms in Spanish for this use case is 39 , being most of them appropriate to the use case (e.g. solucionar, informar, presentar $^{9}$ ). However, other results are not applicable to the use case, such as denunciar (report, in the legal sense of the word).

\subsection{Evaluation}

The results obtained in the 2 use cases above have been evaluated to determine the suitability of the

\footnotetext{
${ }^{9}$ solve, inform and show, respectively.
}

strategy described in this work. For this, a gold standard has been created using expert knowledge, by considering the synonyms in the target language (in this case, Spanish) for all the lexical units corresponding to the selected frames for each use case.

For this evaluation, different combinations of the manual selection steps in Section 3 have been applied, and their corresponding synonyms have been obtained through the resulting synsets. The combinations evaluated are the following:

- Frame selection (F). All lexical units for the selected frames and their associated synonyms.

- Frame + lexical unit selection $(\mathbf{F}+\mathbf{L U})$. Selection of lexical units for the selected frames.

- Frame selection + SUMO filtering (F+SUMO). All lexical units for the selected frames, plus SUMO filtering.

- Frame+lexical unit selection+SUMO filtering ( $\mathbf{F}+\mathbf{L U}+\mathbf{S U M O})$.Selection of lexical units for the selected frames, plus SUMO filtering.It is the strategy defined in this work.

Table 1 shows the number of synonyms obtained in each combination ${ }^{10}$. For each of the configurations, precision-recall-F1 evaluations have been performed, the results of which can be seen in Table 2. These evaluations show that the less filtering, the more recall, as the more synonyms obtained, the more possibility they will include the gold ones. In terms of $\mathrm{F} 1$, on average, unique filtering configurations ( $\mathrm{F}+\mathrm{LU}$ and $\mathrm{F}+\mathrm{SUMO}$ ) obtain practically the same results. It is especially relevant that SUMO filtering is able to obtain on average better precision

\footnotetext{
${ }^{10}$ Data can be found at https://git.io/JB2HY.
} 


\begin{tabular}{lccccccccc} 
& \multicolumn{3}{c}{ Guide } & \multicolumn{3}{c}{ CMMS } & \multicolumn{3}{c}{ Average } \\
\cline { 2 - 10 } & P & R & F1 & P & R & F1 & P & R & F1 \\
\hline F & 0.04 & 1 & 0.07 & 0.12 & 1 & 0.22 & 0.08 & 1 & 0.15 \\
F+LU & 0.13 & 0.88 & 0.23 & 0.23 & 0.94 & 0.37 & 0.18 & 0.91 & 0.30 \\
F+SUMO & 0.14 & 0.76 & 0.23 & 0.21 & 0.88 & 0.34 & 0.25 & 0.82 & 0.29 \\
F+LU+SUMO & 0.50 & 0.64 & 0.56 & 0.28 & 0.64 & 0.39 & 0.39 & 0.64 & 0.48 \\
\hline
\end{tabular}

Table 2: Precision (P), recall (R) and F1 metrics for all configurations for each use case, and average results.

results than LU selection -+0.07 points-, which emphasises the importance of a semantic filtering step in this type of task. Nevertheless, it is important to point out that both configurations help improve the results. In this sense, both processes combined are complementary, since their combination allows to significantly improve the base results obtained with F.

All in all, these results show that manual filtering is necessary in this type of task, as it has been proved that improves up to 0.3 points the $\mathrm{F} 1$ measure on average. Also, the figures obtained allow to determine that this is a complex task that deserves further investigation.

\subsection{Other remarks}

The information gathered in the use cases considered in this work allows to instantiate the domain ontology with intent-detection-relevant data reducing effort and potential intent identification errors. As it can be seen from the data reported in Sections 4.1 and 4.2, the incremental filtering through the defined steps allows to drastically reduce options, and semantic filtering allows to fine-tune the lexical units to be associated to an intent.

As a side note, and regarding both use cases, manual selection is not always a straight-forward process. Regarding frames, the online version of FrameNet ${ }^{11}$-with the corresponding description of each frame in human-friendly terms- may be used to be able to differentiate ambiguous frames.

\section{Conclusions}

This work focuses on the intent detection step in an ontology-based task-oriented dialogue system, which requires a modelling of the intents into the domain ontology. Since the intents and the words that trigger them vary depending on the use case, the task of instantiating the domain ontology with

\footnotetext{
${ }^{11}$ http://sato.fm.senshu-u.ac.jp/ frameSQL/fn2_15/notes/
}

such information every time a new use case is needed is time and cost consuming.

The paper has presented a generic, semiautomatic strategy that reuses existing multilingual lexical resources to assist ontology engineers in modelling the specific intents and the words that elicit them for a given use case. This method consists of a preliminary characterization of the use case, a selection of relevant FrameNet frames, lexical units in English and SUMO tags and an automatic data gathering for intents given the previously selected data. The result is a structured set of intents, their corresponding frames and the words that evoke them. Finally, and depending on the modelling of the target domain ontology, a SPARQL query is generated, to be used to instantiate it.

To prove the generality of the approach, this strategy has been validated in 2 different industrial use cases: interaction in Spanish with a guide robot and with a CMMS. Moreover, an evaluation in terms of precision, recall and F1 has been performed. This evaluation validates the steps described in this work and their positive contribution to instantiate valid intent-related information with a lower resource consumption than doing so manually.

Further work includes research oriented to the improvement of the quality of the data extracted. As stated previously, PM only includes frames that have verbs as lexical units, leaving out other frames, such as Information, that have nominal or adjectival lexical units. In this line, it may be interesting to explore other sources of information that allow to obtain information about these missing frames and in different languages, in a similar manner that PM does with frames with verbal lexical units.

\section{Acknowledgements}

This work was partially supported by the Basque Government's Elkartek R\&I program, projects EKIN (KK-2020/00055) and DeepText (KK2020/00088). 


\section{References}

Javier Alvez, Itziar Gonzalez-Dios, and German Rigau. 2019. Commonsense reasoning using wordnet and sumo: a detailed analysis. arXiv preprint arXiv:1909.02314.

Jordi Atserias, Luis Villarejo, German Rigau, Eneko Agirre, John Carroll, Bernardo Magnini, and Piek Vossen. 2004. The MEANING Multilingual Central Repository. In Proceedings of the 2nd International Global WordNet Conference, Jan 20-23, 2004, pages 23-30. Masaryk University.

Aboubakr Benabbas, Hannes Hornig, and Daniela Nicklas. 2018. Semi-Automatic Ontology Population for Online Quality Assessment of Particulate Matter Sensors. In Intelligent Environments 2018, pages 119-128. IOS Press.

Luisa Bentivogli, Pamela Forner, Bernardo Magnini, and Emanuele Pianta. 2004. Revising the WordNet Domains Hierarchy: Semantics, Coverage and Balancing. In Proceedings of the Workshop on Multilingual Linguistic Resources, pages 94-101.

Christopher M Bishop. 2006. Pattern Recognition and Machine Learning. Springer.

Daniel G Bobrow, Ronald M Kaplan, Martin Kay, Donald A Norman, Henry Thompson, and Terry Winograd. 1977. GUS, a Frame-Driven Dialog System. Artificial Intelligence, 8(2):155-173.

Manon Cassier, Zied Sellami, and Jean-Pierre Lorré. 2019. Meeting Intents Detection Based on Ontology for Automatic Email Answering. In 30es Journées Francophones d'Ingénierie des Connaissances, IC 2019, pages 99-111.

Hongshen Chen, Xiaorui Liu, Dawei Yin, and Jiliang Tang. 2017. A Survey on Dialogue Systems: Recent Advances and New Frontiers. SIGKDD Explor. Newsl., 19(2):25-35.

Qian Chen, Zhu Zhuo, and Wen Wang. 2019. BERT for Joint Intent Classification and Slot Filling. CoRR.

F. Corcoglioniti, M. Rospocher, and Alessio Palmero Aprosio. 2016. Frame-Based Ontology Population with PIKES. IEEE Transactions on Knowledge and Data Engineering, 28:3261-3275.

Corinna Cortes and Vladimir Vapnik. 1995. SupportVector Networks. Machine learning, 20(3):273297.

Maddalen Lopez De Lacalle, Egoitz Laparra, Itziar Aldabe, and German Rigau. 2016. Predicate Matrix: Automatically Extending the Semantic Interoperability between Predicate Resources. Language Resources and Evaluation, 50(2):263-289.

Maddalen Lopez De Lacalle, Egoitz Laparra, and German Rigau. 2014. Predicate Matrix: Extending SemLink through WordNet Mappings. In LREC, pages 903-909.
Charles J Fillmore et al. 1976. Frame Semantics and the Nature of Language. In Annals of the New York Academy of Sciences: Conference on the Origin and Development of Language and Speech, volume 280, pages 20-32.

Ander González-Docasal, Cristina Aceta, Haritz Arzelus, Aitor Álvarez, Izaskun Fernández, and Johan Kildal. 2020. Towards a Natural Human-Robot Interaction in an Industrial Environment, pages 243255. Springer Singapore.

Xavier Gómez Guinovart, Itziar Gonzalez-Dios, Antoni Oliver, and German Rigau. 2021. Multilingual central repository: a cross-lingual framework for developing wordnets. arXiv preprint arXiv:2107.00333.

Arshit Gupta, John Hewitt, and Katrin Kirchhoff. 2019. Simple, Fast, Accurate Intent Classification and Slot Labeling for Goal-Oriented Dialogue Systems.

Daniel Jurafsky and James H. Martin. 2020. Speech and Language Processing (Draft). Hentet.

Paul Kingsbury and Martha Palmer. 2003. Propbank: the Next Level of Treebank. In Proceedings of Treebanks and Lexical Theories, volume 3. Citeseer.

Efstratios Kontopoulos, Panagiotis Mitzias, Marina Riga, and Ioannis Kompatsiaris. 2017. A DomainAgnostic Tool for Scalable Ontology Population and Enrichment from Diverse Linked Data Sources. In DAMDID/RCDL, pages 184-190.

Irina Leshcheva, Evgeny Blagov, and Anastasiia Pleshkova. 2017. Towards a method of ontology population from heterogeneous sources of structured data. In 2017 IEEE 30th Neumann Colloquium $(N C)$, pages 29-34.

Samuel Louvan and Bernardo Magnini. 2020. Recent Neural Methods on Slot Filling and Intent Classification for Task-Oriented Dialogue Systems: A Survey. In Proceedings of the 28th International Conference on Computational Linguistics, pages 480 496, Barcelona, Spain (Online). International Committee on Computational Linguistics.

Jawad Makki. 2017. OntoPRiMa: A prototype for automating ontology population. International Journal of Web/Semantic Technology (IJWesT), 8.

George A Miller. 1995. WordNet: a Lexical Database for English. Communications of the ACM, 38(11):39-41.

Ian Niles and Adam Pease. 2001. Towards a Standard Upper Ontology. In Proceedings of the International Conference on Formal Ontology in Information Systems, pages 2-9.

Adam Pease, Ian Niles, and John Li. 2002. The Suggested Upper Merged Ontology: A large Ontology for the Semantic Web and its Applications. In Working Notes of the AAAI-2002 Workshop on Ontologies and the Semantic Web, volume 28, pages 7-10. 
Abdul Quamar, Chuan Lei, Dorian Miller, Fatma Ozcan, Jeffrey Kreulen, Robert J Moore, and Vasilis Efthymiou. 2020. An Ontology-Based Conversation System for Knowledge Bases. In Proceedings of the 2020 ACM SIGMOD International Conference on Management of Data, pages 361-376.

Eleanor Rosch. 1977. Human Categorisation. Studies in Cross-Cultural Psychology, I(1):1-49.

Karin Kipper Schuler. 2006. VerbNet: A BroadCoverage, Comprehensive Verb Lexicon. Ph.D. thesis, University of Pennsylvania. 